\title{
Light-Induced Self-Writing Effects in Bulk Chalcogenide Glass
}

\author{
Ami M. Ljungström, Member, OSA, and Tanya M. Monro, Member, OSA
}

\begin{abstract}
A waveguide can be self-written by a beam of light propagating in a photosensitive material. We report the first observation of self-writing effects in bulk chalcogenide glass and investigate the influences of different writing beam sizes and powers. We observe increases in refractive index of $2.5 \times$ $10^{-4}$ due to illumination at $1047 \mathrm{~nm}$ in Ce-doped Ga-La-S. Simulations of the self-writing process show a good agreement with the experimental results. This verifies our numerical model and allows the dynamics of this process to be explored. Using this knowledge, we predict the experimental parameters and conditions required to write waveguides, tapers, and ultimately complex three-dimensional (3-D) structures.
\end{abstract}

Index Terms-Chalcogenide glass, channel, photosensitivity, self-writing, taper, waveguide.

\section{INTRODUCTION}

$\mathbf{T}$ HIS paper explores self-writing processes in which a beam of light induces refractive index changes that dynamically evolve to form a waveguide, which then guides this light [1]. To perform self-writing, a photosensitive material must be used; materials are described as photosensitive if they experience a long-lasting refractive index change when exposed to light. We begin by briefly illustrating this concept, which is described in detail in [1]-[4].

Consider a Gaussian beam focused on a material with an initially uniform refractive index. If the material is photosensitive at the writing wavelength, this diffracting light distribution starts to change the index within the material. The largest refractive index changes occur at positions where the light intensity is highest, which, in this case, is along the propagation axis. If the index increases in response to illumination, this change acts to reduce the diffraction of the incoming beam. In these early stages, diffraction imprints an adiabatic taper within the material, and, over time, this taper can evolve into a channel waveguide, guiding the writing beam through the material.

This process is called self-writing because the same beam that creates the waveguide is subsequently guided by it. After it has formed, a self-written waveguide can be used to guide light at other wavelengths. As these changes in index can be permanent and local, this process is distinct from other self-action effects, such as spatial and photorefractive solitons.

Waveguides are currently formed using many different techniques, including epitaxial growth, diffusion methods,

Manuscript received February 22, 2001; revised June 6, 2001. The work of T. M. Monro was supported by a Royal Society University Research Fellowship. The authors are with the Optoelectronics Research Centre, University of Southampton, Southampton SO17 1BJ, U.K. (e-mail: aml@orc.soton.ac.uk). Publisher Item Identifier S 0733-8724(02)00382-1. and direct-writing [5]. The direct-writing technique has the advantage that using photosensitivity avoids the complex lithographical steps used in the other techniques. However, in direct writing, either the sample or the beam must be translated to pattern the waveguide. In contrast, using self-writing, the waveguide evolves dynamically, and so it is a one-step process that requires no translation. Theoretical work [2] shows that the form of the resulting waveguide can be tailored by appropriate choice of writing beam shape, which allows the formation of a wide range of complex structures. In addition, because self-written waveguides evolve dynamically, they vary adiabatically along their length, and so should experience minimal radiation losses due to the absence of sharp bends. This is a relatively new area of research and in this paper only channel waveguides are considered. The evolution of self-written waveguides in bulk glasses is a rich new area of physics that has not yet been explored.

Self-writing can be realized in a variety of photosensitive materials; what is required is a long-lasting change in refractive index in response to illumination. Previously, self-written channel waveguides have been created in both planar germanosilicate glass [3] and $\mathrm{As}_{2} \mathrm{~S}_{3}$ chalcogenide glass [6]. This process has also been studied experimentally in bulk geometry, in which solid waveguides have been formed in a liquid photopolymer [7], tapers have been formed in ultraviolet (UV)-cured epoxy [8], and, recently, a three-dimensional (3-D) photonic crystal was fabricated by self-writing channels in a photopolymerizable resin [9], [10]. The fact that self-writing has been carried out in such a wide range of materials demonstrates that it is a robust process that can occur regardless of the precise chemical or structural mechanisms responsible for the index change.

Simulations of the self-writing process completed to date have principally considered the planar geometry, which is less computationally demanding than the bulk case. These studies have been performed both for glass [1] and liquid photopolymers [7]. Some preliminary simulations for bulk glasses have also been done, which show that a channel waveguide can form in a bulk material provided that a sufficiently large refractive index change is possible in the material [4].

In this paper, we explore self-writing in bulk glass, both experimentally and theoretically. Self-writing has not previously been experimentally demonstrated in any bulk glass. The bulk geometry offers greater flexibility than the planar geometry to form complex, 3-D waveguides. In addition, glasses offer the advantage of straightforward integration with conventional glass technologies. Most photosensitive glasses are fabricated using plasma-enhanced chemical-vapor deposition (PECVD) 
[11] or flame hydrolysis techniques [12], whereas the bulk glasses we use require no such complex processing.

\section{MATERIAL}

In these experiments, a compound chalcogenide glass, gallium lanthanum sulphide ( $\mathrm{Ga}-\mathrm{La}-\mathrm{S}, \mathrm{n}=2.5[13])$, is used. This family of glasses typically has the potential for large photosensitive index changes under illumination [13], although this photosensitivity is not yet well characterized. The properties of $\mathrm{Ga}-\mathrm{La}-\mathrm{S}$ can be altered somewhat by doping the glass, and in our experiments, pure $\mathrm{Ga}-\mathrm{La}-\mathrm{S}, \mathrm{Ga}-\mathrm{La}-\mathrm{S}$ oxide, and cerium-doped $\mathrm{Ga}-\mathrm{La}-\mathrm{S}$ have been used. We find that the biggest index changes are achieved in the cerium-doped sample. These results are presented here. The composition of this glass is $70 \mathrm{Ga}_{2} \mathrm{~S}_{3}: 27.5 \mathrm{La}_{2} \mathrm{~S}_{3}: 1 \mathrm{La}_{2} \mathrm{O}_{3}: 1.5 \mathrm{Ce}_{2} \mathrm{~S}_{3}$, which corresponds to a doping concentration of $1.5 \mathrm{~mol} \%$ cerium.

Light with a wavelength near the band edge typically induces changes in a material, because significant absorption occurs here, and, for $\mathrm{Ga}-\mathrm{La}-\mathrm{S}$, the band edge is located near $500 \mathrm{~nm}$ [14]. Therefore, one might expect that a two-photon absorption process would lead to photosensitivity around $1 \mu \mathrm{m}$. This wavelength is preferable because losses through the material are lower here than near the band edge. In our experiments, a diode-pumped Nd-YLF fiber-coupled laser $(1.047 \mu \mathrm{m})$ is used to illuminate the sample. For these experiments, a high spatial quality of the writing beam is required and, therefore, fiber lasers are favorable. Also, the input and output faces of the sample are both polished in order to obtain parallel high-quality faces.

\section{EXPERIMENT}

In these experiments, a Gaussian beam is focused down to a waist on the input edge of a 5-mm-long sample using a lens. Another lens is used to image the output beam onto a beam profiler. The evolution of the index change is observed by monitoring the beam shape at the output face of the sample. Alignment is crucial in this experiment, and good care must be taken to position the waist on the sample edge; it is also important not to preexpose the sample to light.

Initially, the writing beam diffracts freely in the uniform bulk material. If the refractive index of the glass increases in response to light, then we expect the beam at the output edge of the sample to narrow and become more intense as the light begins to be guided by the index change it induces. Fig. 1 shows this evolution for a typical experiment; the change in beam shape confirms that self-writing has begun to occur in the material.

Fig. 2 shows the full-width at half-maximum (FWHM) and the peak intensity of the output beam as functions of time for two different experiments. In both of these experiments, a writing beam of $9 \mu \mathrm{m}$ and a power of $650 \mathrm{~mW}$ are used. First, we concentrate on Fig. 2(a), which correspond to the output beams shown in Fig. 1. It can clearly be seen how the beam narrows and the peak intensity increases during the exposure (both by a factor of 1.5). During this exposure, the loss does not change significantly (the power decreases by $4 \%$ ).

Unlike the experiment in Fig. 2(a), in Fig. 2(b), the FWHM and the peak intensity do not change by the same degree, and

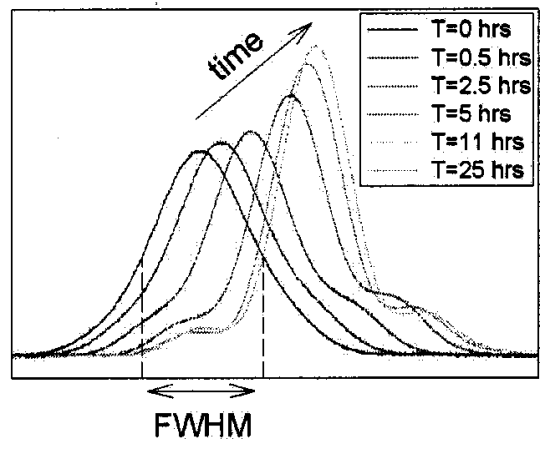

Fig. 1. The shape of the beam emerging from the sample. The beam narrows and becomes more intense during the exposure.

the intensity even starts to decrease toward the end of the exposure. One explanation for this difference is that these samples are somewhat inhomogeneous due to crystalization within the glass. We have confirmed the presence of inhomogeneity by performing both beam quality and loss measurements. Even for the experiment in Fig. 2(a), the beam becomes slightly nonGaussian over time, as shown in Fig. 1.

Approximately $7 \mathrm{~h}$ after the start of the exposure, the FWHM levels off and stops changing. It seems likely that, at this point, the saturation value of the refractive index has been reached and the material cannot change any further; this behavior is discussed later.

The longevity of this change in refractive index has been investigated by monitoring the sample a number of times after the initial exposure, as shown in Fig. 3. It can be seen that the index changes are long lasting and do not decay significantly when the light is removed, at least over the time scale of a day. However, over a longer time scale of a few days, the changes are observed to decay. In future experiments, it may be possible to make these changes permanent by annealing the glass after the initial exposure.

For a given writing beam, the structure that evolves in the material is principally determined by the maximum change in refractive index that can be induced. We show that this necessitates a careful choice of the writing beam size. To obtain significant diffraction, at least a few Rayleigh ranges must fit within the material. However, very narrow beams cannot be used, because a prohibitively large refractive index change is then needed to counteract their diffraction. In the other extreme, the relatively large Rayleigh range of a wide beam results in a need for long samples. This is not only impractical for devices, but, in addition, losses in the material then become problematic. In practice, for any particular material, there exists an optimum choice of experimental parameters. In order to explore how self-writing depends on the writing beamwidth and power, a range of experiments was conducted, as shown in Fig. 4. These experiments correspond to 10-20 Rayleigh ranges within the 5-mm-long sample.

First, we consider the changes in FWHM. Notice that, for this case, the best result was achieved by the experiment shown in Fig. 2(a), using a writing beamwidth of $9 \mu \mathrm{m}$ and a power of $650 \mathrm{~mW}$. It can be seen that there exists a complex relationship between the writing-beam size and power and the resulting degree to which the beam is guided by the index distribution written in the sample. These trends are explored here. 


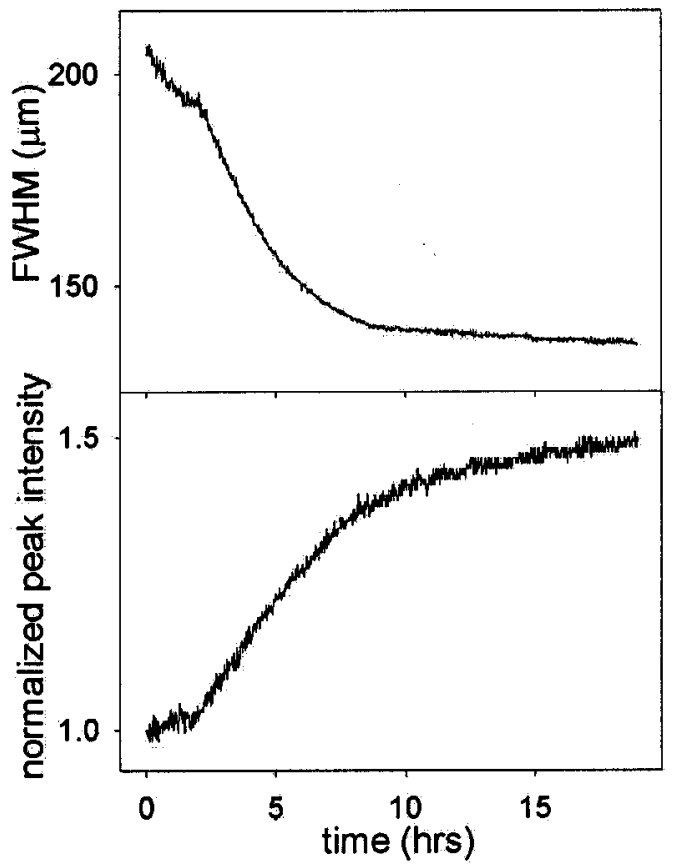

(a)

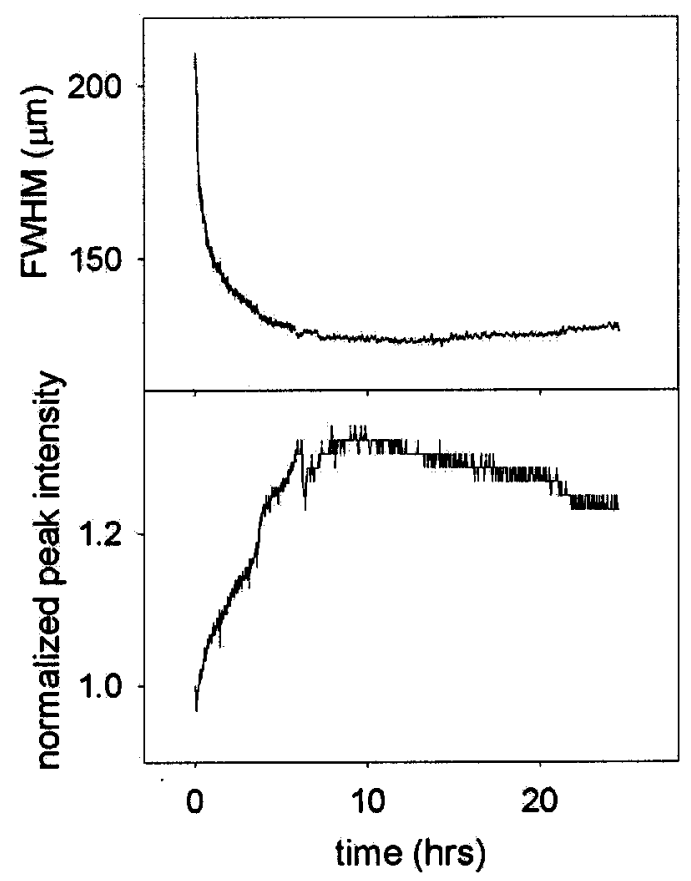

(b)

Fig. 2. The FWHM and the peak intensity of the output beam from two different experiments using a writing beamwidth of $9 \mu \mathrm{m}$ and a power of $650 \mathrm{~mW}$.

Fig. 4 shows that narrow beams and high power together result in bigger overall changes. Hence, we observe that high light intensities cause slightly greater index changes in the material that allow self-writing to proceed further. For wider beams, although bigger changes are achieved when high powers are used, the results are not as sensitive to the power as for narrower beams. Because wider beams require a higher power in order to achieve the same light intensity, in order to get more dramatic changes in this material, the power would have to be increased significantly.

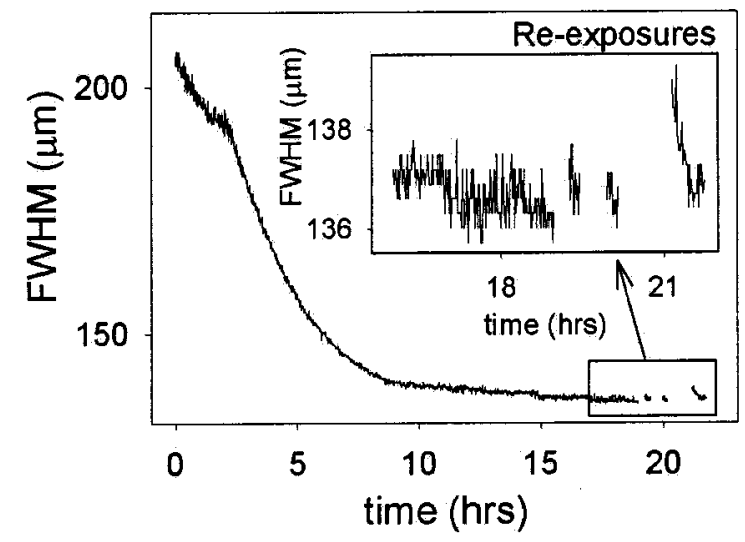

Fig. 3. Measurements of the width of the outcoming beam after the initial exposure [corresponding to Fig. 2(a)]. The changes within the material are long lasting.

Some experiments carried out using high-intensity beams, i.e., $9.5-\mu \mathrm{m}$ beam and $650 \mathrm{~mW}$, resulted in only minor overall changes (these are not included in Fig. 4). We believe that when these high intensities see imperfections on the sample edge, in these cases, damage to the edge occurs. This damage prevents a waveguide from forming, hence, it results in smaller changes. In conclusion, although we find that self-writing proceeds further at high intensities, in such cases, damage of the sample is more likely; thus, more consistent results are obtained using a slightly wider beam.

It can also be seen in Fig. 4 that the FWHM and the peak intensity do not always change by the same amount. The peak intensity is particularly dependent on the loss in light intensity in the material. Due to sample inhomogeneities, the sample loss varies somewhat with position, and, in addition, the loss may change during the exposure, a behavior that has been observed previously in planar glass [2]. In Fig. 4, only a selection of experiments are presented, which show reasonable losses in intensity, good-quality beams, and no damage to the sample.

We observe that self-writing proceeds faster when the light intensity is higher (i.e., a narrow beam with high power produces the fastest change). This observation is consistent with theoretical models for photosensitivity [7], [15]. However, the inhomogeneities in the sample make it hard to interpret the rate of change in refractive index.

These experiments unambiguously demonstrate self-writing effects in these glasses and help to ascertain the parameters that are most critical in this process. In the experiments carried out here, a typical change in FWHM is of a factor of 1.5, when using a $9-\mu \mathrm{m}$-wide beam in a 5-mm-long sample. However, to form a mature channel waveguide, much larger changes are typically expected (see Section V). Next, we use simulations in order to predict how to increase the magnitude of the self-writing effect in bulk glass.

\section{SimUlations}

In order to obtain a deeper understanding of the dynamics of the self-written evolution and to predict the effects of using different experimental parameters, we have conducted numerical simulations of this process. 


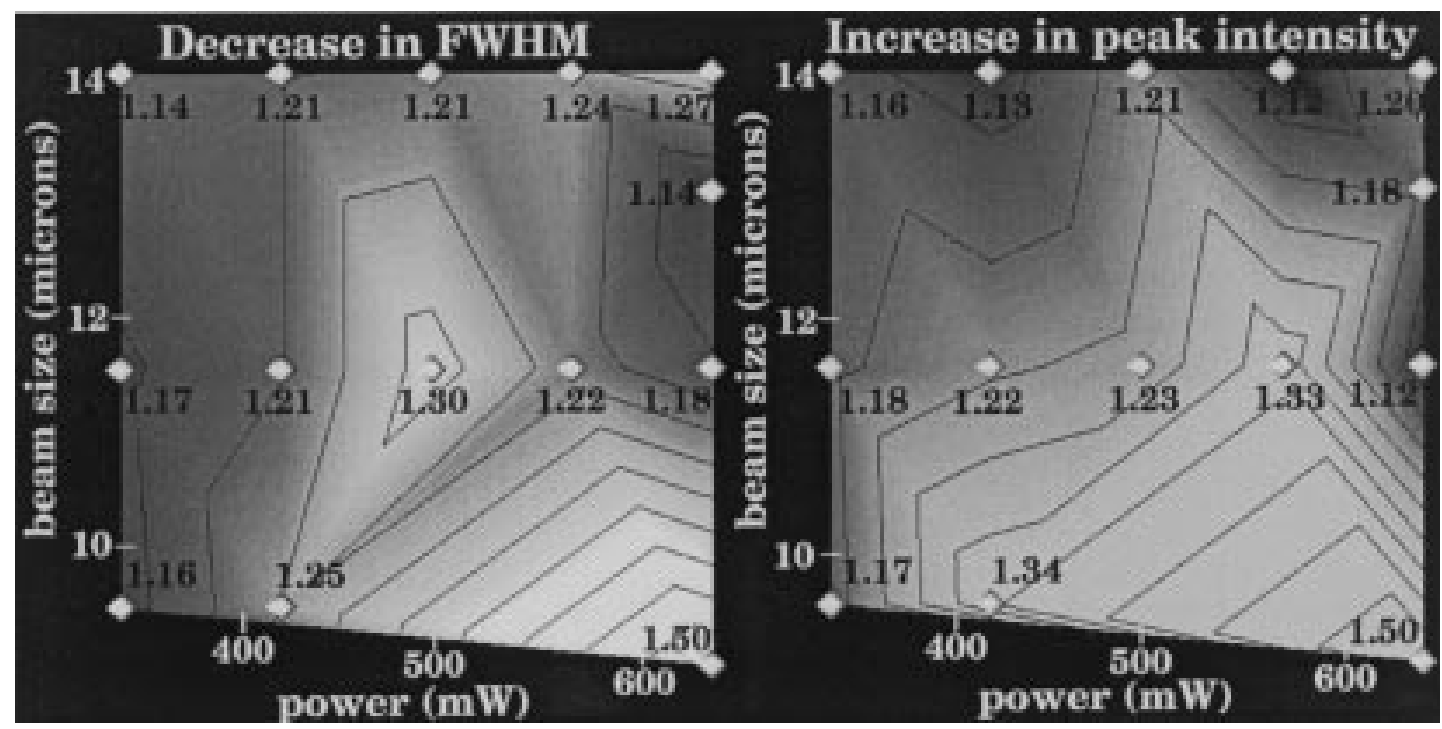

Fig. 4. Summary of experiments carried out using different writing-beam sizes and powers.

A Gaussian beam is used as the writing beam. Thus, the initial condition at the input face $(z=0)$ is

$$
E(x, y, 0, t)=E_{0} \exp \left(-\frac{x^{2}+y^{2}}{a^{2}}\right) .
$$

Here, $E=E(x, y, z, t)$ is the electric field envelope amplitude, $z$ is the propagation direction, and $x$ and $y$ are the transverse coordinates. The beamwidth at the input face is $a$, which corresponds to an FWHM in the intensity of $a \sqrt{2 \ln 2}$. Two differential equations can be used to describe self-writing [1]; the paraxial wave equation describes the propagation of light through the material

$i k_{0} n_{0} \frac{\partial E}{\partial z}+\frac{1}{2}\left(\frac{\partial^{2}}{\partial x^{2}}+\frac{\partial^{2}}{\partial y^{2}}\right) E+k_{0}^{2} n_{0} \Delta n E+\frac{i}{2} k_{0} n_{0} \alpha E=0$

whereas the photosensitivity equation describes the refractive index evolution

$$
\frac{\partial \Delta n}{\partial T}=I^{p}\left(1-\frac{\Delta n}{\Delta n_{s}}\right) .
$$

Here, $n_{0}$ is the initial refractive index, $k_{0}=2 \pi / \lambda$ is the wavenumber, and the loss in decibels per unit length is 4.343 $\alpha$. The intensity $I$ is defined as $E E^{*}$, and $p$ is the number of photons involved in the process (typically 1 or 2 ). Here, $T$ is a normalized time defined as

$$
T=a^{2} k_{0}^{2} n_{0} A_{p} I^{p} t
$$

where $A_{p}$ is a real coefficient that depends on $p$, the material properties, and $\lambda$, and $t$ is the time in seconds.

In (3), a saturation index $\Delta n_{s}$ is introduced to limit the change in index that can occur, and in this model, the index change slows down exponentially as it approaches this value. This simple physical model has been shown to agree well with experiments in photopolymers [7] and germanosilicate glass [3]. It is also consistent with our observation that the process proceeds faster at high light intensities. However, this simple model cannot be completely accurate, because we observed that the degree of index change is somewhat intensity dependent. In bulk geometry, $\Delta n_{s}$ is crucial because the incoming light is focused in two transverse directions, resulting in a high intensity and, hence, a larger change in refractive index. Indeed, it has been shown previously that a saturation index must be included to prevent catastrophic collapse of the beam [4].

The propagation of light through a material must, in general, be described using the vector wave equation [16]. However, if the beam properties vary only slowly in the propagation direction, the paraxial approximation can be used, as given by (2). The Gaussian beam considered here propagates along the $z$ axis; thus, this approximation is valid as long as the diffraction of the beam is not too large. In other words, there exists a minimum beam size $a \gg \sqrt{2} /\left(k_{0} n_{0}\right)$ that is always satisfied here.

To solve the equations describing the self-writing process, advantage is taken of the difference in time scales for the processes involved. Typically, the light propagation occurs in nanoseconds, whereas the evolution of the refractive index takes minutes or hours. Therefore, (2) and (3) can be treated as two independent processes that can be solved separately. This is done numerically on a grid inside the material using a split-step beam-propagation model. Note that, for the bulk geometry, these calculations are computationally intensive, and no simulations have previously been done using real parameters and conditions for these compound glasses. Typical calculations use grids ranging from $128 \times 128 \times 500$ to $256 \times 256 \times 600$, which requires about $0.1-2$ Gb computer memory; these calculations take between days and weeks to run on a 1-GHz processor.

To compare the simulation results with experimental data, the time in the simulation must be scaled, because a normalized time $T$ is used in the model and the parameter $A_{p}$ is unknown [recall (4)]. In these materials, the index change that can be achieved by illumination $\left(\Delta n_{s}\right)$ depends critically on the glass composition and is typically in the range $0.0001-0.001$ [11]. Therefore, within this range, we choose $\Delta n_{s}$ to fit the experimental data.

In Fig. 5, experimental results from Fig. 2(b), using a writing beam size of $9 \mu \mathrm{m}$, are shown together with the results from the corresponding simulation. This experiment was chosen be- 


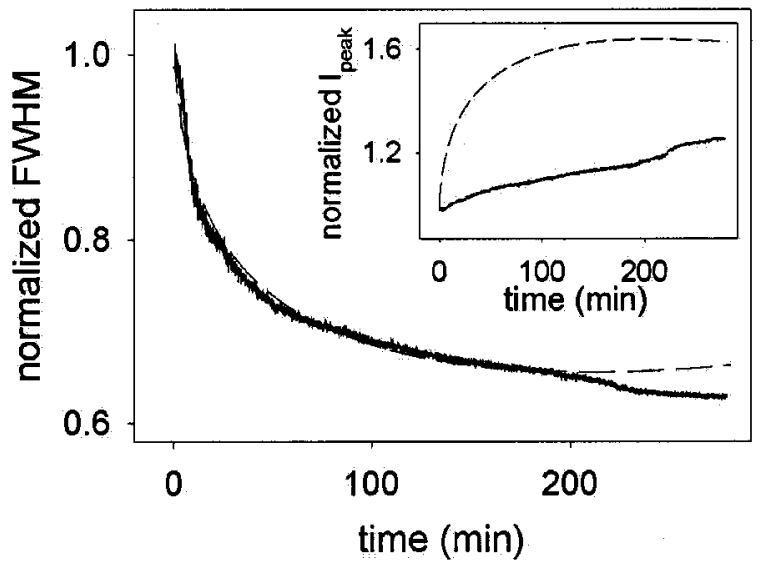

Fig. 5. Change in FWHM for experimental data (FWHM $=9 \mu \mathrm{m}$, solid lines) and simulation using a $\Delta n_{s}$ of $2.5 \times 10^{-4}$ (dashed lines). The inset shows the corresponding changes in peak intensity.

cause it demonstrates a smooth change in FWHM, which should make it easier to compare with theory. In this particular simulation, we obtain good agreement for the shape of the width evolution when $\Delta n_{s}$ is $2.5 \times 10^{-4}$, which is a physically reasonable value. It can be seen that the experimental result and the simulation start to differ somewhat at later times (for $t>200 \mathrm{~min}$ ), which is caused by either inhomogeneities in the sample or our simplified model for the index change; these discrepancies accumulate over time.

The inset in Fig. 5 shows that, although the simulation accurately predicts the behavior of the FWHM, the change in peak intensity differs significantly between experiment and simulation. As mentioned earlier, it has previously been shown that loss has greater influence on the peak intensity than on the FWHM [17]; thus, simulations including loss were undertaken. Initially, the simplest case of constant loss was considered, but agreement with observations could not be improved significantly. Next, the model was extended to include a variable exposure-dependent loss in which the local value of the loss increases in response to changes in the refractive index, as in [17].

Fig. 6 shows again the experimental and simulation data from Fig. 5, together with results from a simulation including this variable loss, represented by solid, dashed, and dotted lines, respectively. The best agreement was found using a variable loss of $6.5 \times 10^{4} \Delta n \mathrm{~dB} / \mathrm{cm}$, which corresponds to a maximum loss of $6.5 \mathrm{~dB} / \mathrm{cm}$ at localized positions where the saturation value of the refractive index has been reached. It can be seen that, although the magnitude change in intensity can be matched by introducing this variable loss, the shape of the evolution differs significantly. Also, the agreement with experiment for the FWHM is reduced. Clearly, a more sophisticated model is needed to get a good agreement between experiment and simulation when a large absorption loss is present.

The shape of the beam emerging from the sample often changes slightly during the exposure, as in Fig. 1, where the beam grows a shoulder at late times in the experiment. Because the writing beam is Gaussian at the input face and the self-written structure is single moded (see Section V), this asymmetry must be caused by some inhomogeneity in

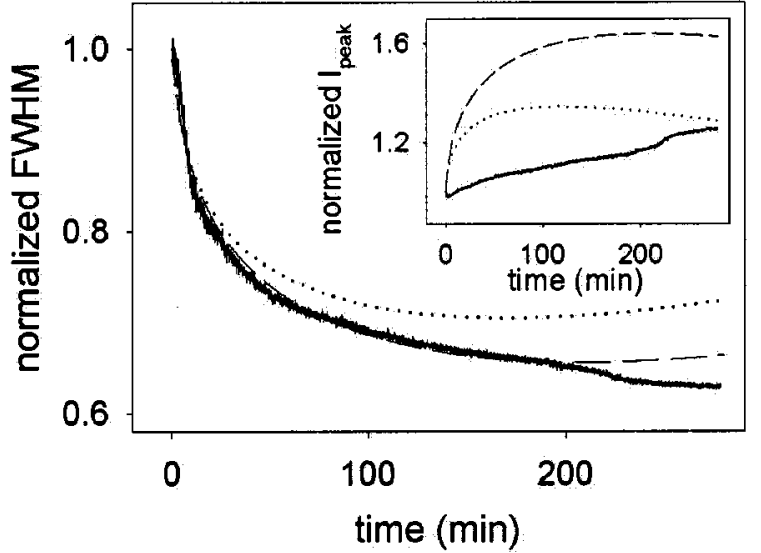

Fig. 6. FWHM at the sample output edge from experiment (solid lines) and simulations. The change in peak intensity is shown in the inset. Dashed curves represent results without loss and dotted lines include a variable exposure-dependent loss of $6.5 \times 10^{4} \Delta n \mathrm{~dB} / \mathrm{cm}$.

the sample; indeed, we find that, in some experiments, the beams retain their Gaussian shape throughout the evolution. In addition, we find that the shape of the beam is not particularly sensitive to the launch conditions, which is further evidence of single-modedness. Because the model used here assumes that the material is homogeneous, we cannot expect perfect agreement with the experimental data, especially at late times.

Clearly, the model used in these simulations captures the essential physics of these self-writing experiments. Next, we use the experimental parameters deduced from this approach to achieve a deeper understanding of the self-written evolution occurring inside the material.

\section{ANALYSIS}

It has been shown previously [1] that, in order to form a channel waveguide, the refractive index at the input face must grow large enough to counteract the diffraction of the beam and focus the light in to an intensity maximum inside the material. This maximum is referred to as the primary eye and has, so far, always been viewed as a precursor to waveguide formation. Typically, this eye is located near the input face so that series expansions can be used to explore its behavior at small distances in the propagation direction $z$. This method has been applied to the planar geometry for $p=1$ and 2 and in bulk for $p=1$ [2], corresponding to bulk photopolymer experiments from [7]. Here, we extend this treatment to correspond to the two-photon absorption process in bulk cerium doped $\mathrm{Ga}-\mathrm{La}-\mathrm{S}$ glass with the aim of achieving a greater understanding of our experimental results.

At early times, the refractive index is too small to focus the incoming beam; thus, a primary eye cannot form and the beam simply diffracts. We find, from the series expansions, that, in our case, the primary eye forms at time $T=T_{0}$ [as defined in (4)] that satisfies

$$
\frac{\ln \left(4 T_{0}\right)}{T_{0}}=\frac{2 n_{0}}{k_{0}^{2} a^{2} \Delta n_{s}}
$$




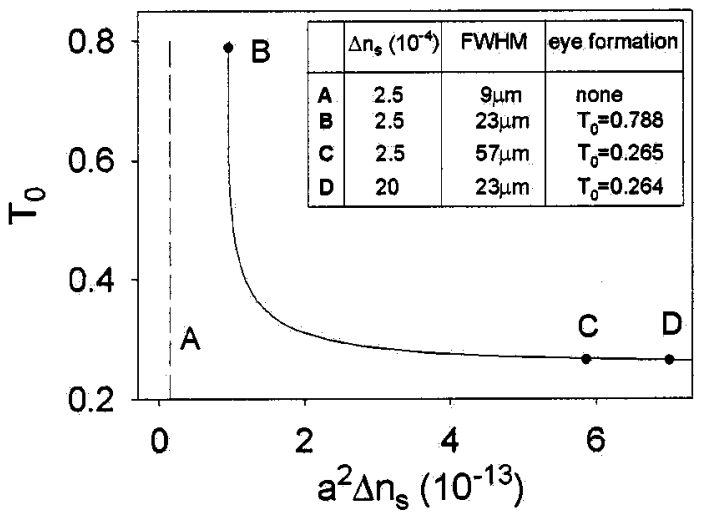

Fig. 7. The primary eye formation time $\left(T_{0}\right)$ as a function of beamwidth and $\Delta n_{s}$. Dots show the formation time for four different choices of experimental parameters.

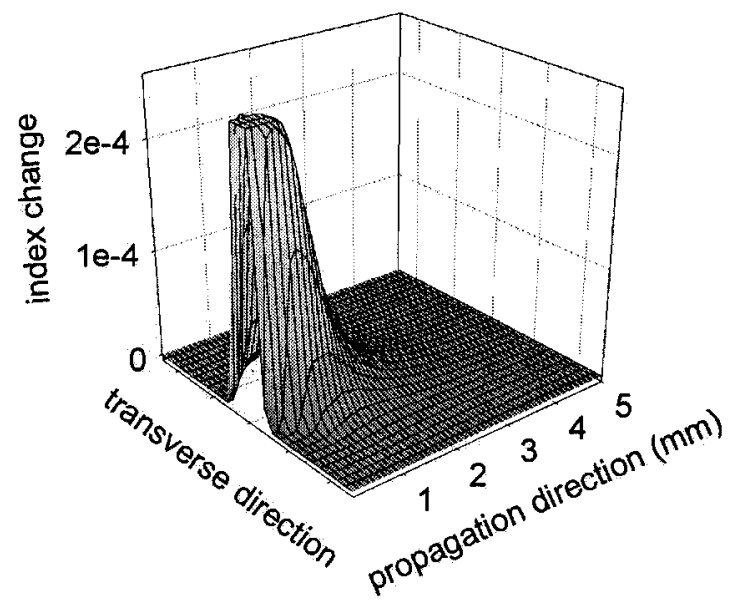

Fig. 8. Case A. The refractive index distribution within the material using a writing beam size of $9 \mu \mathrm{m}$ and $\Delta n_{s}=2.5 \times 10^{-4}$. This corresponds to the simulation shown in Fig. 5 at $t=195$ min.

For further details about this approach, see [2].

From (5), it can be seen that, for any specific $\Delta n_{s}$, there exists a minimum writing beamwidth for an eye to form. If the beam is too narrow, the material cannot ever change index enough to counteract its diffraction. For the glass in our experiment, $n_{0}$ is approximately 2.5 , and a good agreement with experiments occurs when $\Delta n_{s}$ is taken to be $2.5 \times 10^{-4}$ (see Section IV). Using these values, (5) is plotted in Fig. 7 and the times at which the primary eye forms with four different choices of experimental parameters are marked A-D. Each of these cases is discussed here.

1) Case A: This case corresponds to the experiments in Fig. 2. It can be seen in Fig. 7 that the primary eye can never form using this beamwidth; therefore, a waveguide will not form. This is verified in Fig. 8, where the contour plot of the index distribution inside the material is plotted late in the evolution ( $t=195 \mathrm{~min}$ in Fig. 5). Here, the saturation value has been reached near the input face. Observe from Fig. 8 that the index change penetrates $1 \mathrm{~mm}$ into the material and, thus, no waveguide is formed. This resulting structure functions as an adiabatic taper, reducing the diffraction of the input beam.

Note that, at the input face, the waveguide width is approximately $12 \mu \mathrm{m}$ and $\Delta n=2.5 \times 10^{-4}$, so the waveguide pa-

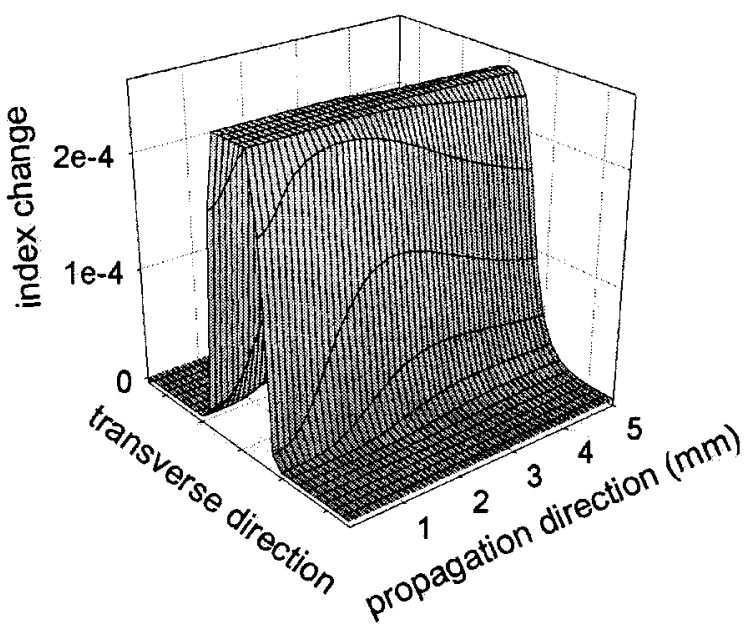

Fig. 9. Case B. The refractive index distribution when a beam of $23 \mu \mathrm{m}$ is used. Here, a $\Delta n_{s}$ of $2.5 \times 10^{-4}$ is enough for a channel to form through the material. However, this will require a very long time; here, $t=740 \mathrm{~min}$.

rameter $V \approx 1.3$ [18] (assuming a step-index profile), which implies that the structure is rigorously single moded. The structure becomes somewhat wider further into the material and, at its widest, $V \approx 2.8$ (width $=26 \mu \mathrm{m}$ ), so that, at most, two modes would be supported. However, because the taper is single moded at the input face, where the beam is launched, it is effectively single moded throughout the material.

2) Case B: Leaving the saturation index the same as in case A, but increasing the beamwidth to $23 \mu \mathrm{m}$, (5) can be satisfied and an eye can form. However, this will take a relatively long time; $T_{0}=0.788$ in Fig. 7. By using (4), the real physical time corresponding to this $T_{0}$ can be determined. For practical reasons, we assume that, when a different width is chosen, the total power in the writing beam remains unchanged. Hence, we scale $t$ in (3) by $\left(2 p / \pi a^{2}\right)^{p}(p=2$ here), and the primary eye forms after 8 min. In Fig. 9, the refractive index distribution that evolves inside the material using these parameters is shown at $t=740 \mathrm{~min}$. It can be seen that this wider beam has allowed the structure to penetrate through the material.

Note that, here, $V \approx 7$, and, thus, the waveguide is multimoded. It has been shown, previously, that self-written structures typically become multimoded at the writing wavelength before a mature channel forms [1]. However, once written, the channel can guide light at other wavelengths, and at longer wavelengths it can be single moded. Although a channel can form using this beamwidth, the primary eye has a very long formation time, and, thus, a mature waveguide takes a long time to evolve, which is not ideal.

3) Case C: Here, the beamwidth has been increased even further to $57 \mu \mathrm{m}$. For this case, the time at which the primary eye forms is $T_{0}=0.265$ (16 min using the same power as in Case B). This would be the ideal experiment using cerium-doped Ga-La-S, where the saturation index, of course, is fixed. In Fig. 10, the refractive index distribution is shown when a mature channel has formed, $t=1500 \mathrm{~min}$. Although the channel takes longer to evolve, here, $V \approx 3$ and, thus, the channel will be single moded at shorter wavelengths than in Case B. Recall that, in our simplified model, the same change in refractive index 


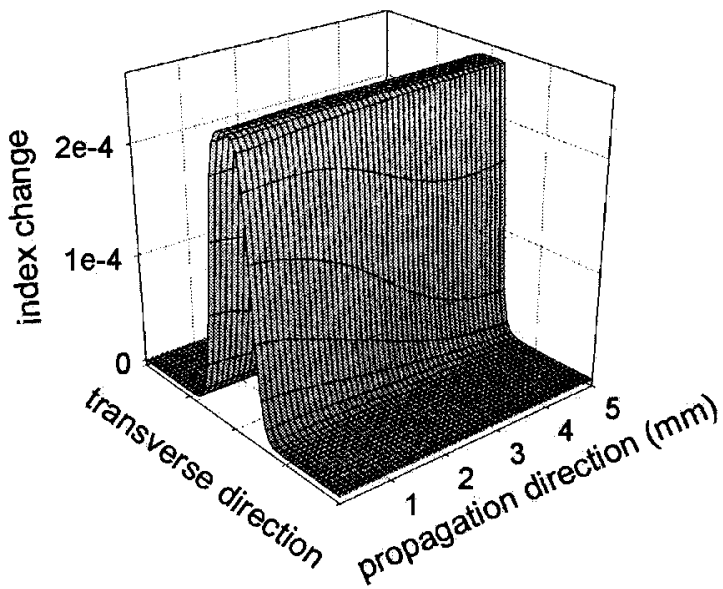

Fig. 10. Case $\mathrm{C}$. The refractive index distribution inside the material when a writing beam of $57 \mu \mathrm{m}$ is used. Here, $t=1500 \mathrm{~min}$ and a mature, uniform channel is formed.

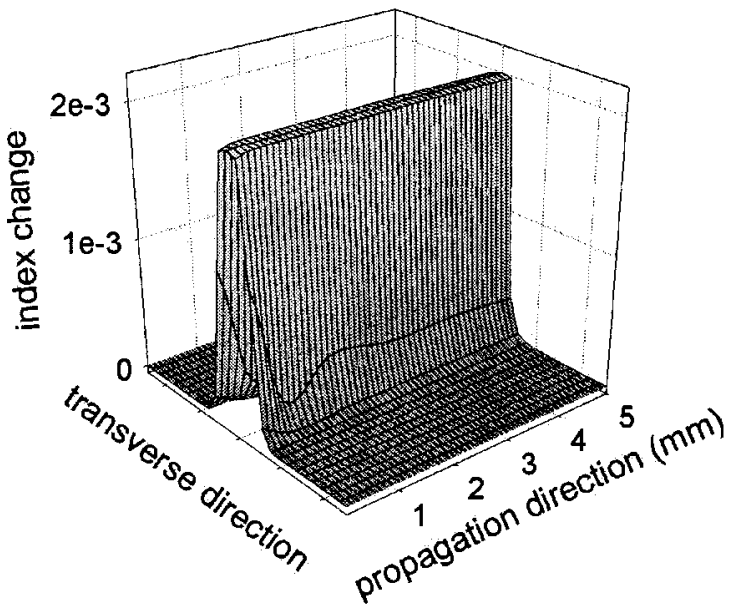

Fig. 11. Case D. The refractive index distribution when $\Delta n_{s}=2 \times 10^{-3}$ and a writing beam of $23 \mu \mathrm{m}$ is used, which results in the formation of a mature channel waveguide. Here, $t=318 \mathrm{~min}$.

will ultimately occur, regardless of the intensity. However, we see from Fig. 4 that this is not strictly true for this material, and that, in order to use such a wide beam, the power needs to be increased.

4) Case D: Another way to form a mature channel is to use a material with a larger saturation refractive index. For example, if $\Delta n_{s}=2 \times 10^{-3}$, the formation time will be $T_{0}=0.264$ ( $3 \mathrm{~min}$ ) using a beamwidth of $23 \mu \mathrm{m}$ (see Fig. 7). Fig. 11 shows that a mature uniform channel waveguide has formed by $t=$ $318 \min (V \approx 10)$. Not surprisingly, a large refractive index increase is favorable because it makes the self-writing proceed more quickly. Recall that, in these simulations, the speed of the process scales with the writing beam power. For example, if twice the power were used here, the waveguide would evolve four times faster. In this simulation, the FWHM decreased by a factor of 9, which is significantly larger than the change of 1.5 that was observed in the experiments shown in Fig. 2. This saturation index $\left(2 \times 10^{-3}\right)$ is achievable in other materials, and, thus, this experiment should be practical in the future.
Information about the primary eye can be used to predict the parameters required to form a channel waveguide. It explains why a mature channel did not form in our experiments, in which the narrow beams used prevent the primary eye from forming. In addition, it helps to determine which materials should be most suitable for future experiments.

\section{DisCUSSION AND CONCLUSION}

These experiments represent the first observations of selfwritten effects in a bulk glass. The refractive index within the cerium-doped $\mathrm{Ga}-\mathrm{La}-\mathrm{S}$ glass increased under illumination and, thus, the diffraction of the propagating beam was decreased. By increasing the beam intensity, a significant change in index occurred and the process progressed faster. Numerical simulations of the process show good agreement with the experiments, which validates the model and indicates that we understand the basic principles of the process. Using this modeling, we have shown that self-written channel waveguides can form in these bulk materials using appropriate parameters and conditions.

To obtain deeper physical insight into our experimental results, analytical series expansions have been used. The behavior of the primary eye was explored, and, hence, the formation of waveguides using different experimental parameters was analyzed. It was found that using narrow beams $(<\sim 20 \mu \mathrm{m})$, as in the experiments with cerium-doped $\mathrm{Ga}-\mathrm{La}-\mathrm{S}$ glass, the refractive index change in the sample, $\Delta n_{s}=2.5 \times 10^{-4}$, was not large enough to form an eye and thus create a waveguide through the material. Despite this limitation, the diffraction of the beam propagating through the material decreased significantly during the experiment and a weak taper was formed.

By introducing different dopants, the photosensitivity of these glasses can be improved, which would enable bigger refractive index changes and, therefore, more sophisticated structures. Because these self-written structures are typically long lasting, waveguides in these glasses could be subsequently used to guide light at other wavelengths. Such compound glass materials transmit light in the far infrared from 0.5 to $10 \mu \mathrm{m}$ [19]; thus, long-wavelength devices could be formed using this approach.

Although, in this early work, only Gaussian writing beams are considered, theoretical work shows that the shape of the resulting waveguide can be tailored via the writing beam [2]. This 3-D bulk geometry should enable great flexibility for creating complicated waveguide structures.

\section{ACKNOWLEDGMENT}

The authors would like to thank Dr. D. Hewak and A. Mairaj from the Optoelectronics Research Centre, University of Southampton, for fabrication of the samples and for helpful discussions.

\section{REFERENCES}

[1] T. M. Monro, C. M. de Sterke, and L. Poladian, "Investigation of waveguide growth in photosensitive germanosilicate glass," J. Opt. Soc. Amer. $B$, vol. 13, pp. 2824-2832, Dec. 1996.

[2] - "Analysis of self-written waveguides in photopolymers and photosensitive materials," Phys. Rev. E, vol. 57, pp. 1104-1113, Jan. 1998. 
[3] T. M. Monro, D. Moss, M. Bazylenko, C. M. de Sterke, and L. Poladian, "Observation of self-trapping of light in a self-written channel in photosensitive glass," Phys. Rev. Lett., vol. 80, pp. 4072-4075, May 1999.

[4] T. M. Monro, C. M. de Sterke, and L. Poladian, "Catching light in its own trap," J. Mod. Opt., vol. 48, pp. 191-238, 2001.

[5] M. Svalgaard, C. V. Poulsen, A. Bjarklev, and O. Poulsen, "Direct UV writing of buried singlemode channel waveguides in Ge-doped silica films," Electron. Lett., vol. 30, pp. 1401-1403, Aug. 1994.

[6] C. Meneghini and A. Villeneuve, " $A s_{2} \mathrm{~S}_{3}$ photosensitivity by two-photon absorption: holographic gratings and self-written channel waveguides," J. Opt. Soc. Amer. B, vol. 15, pp. 2946-2950, Dec. 1998.

[7] A. S. Kewitsch and A. Yariv, "Self-focusing and self-trapping of optical beams upon photopolymerization," Opt. Lett., vol. 21, pp. 24-26, Jan. 1996.

[8] S. J. Frisken, "Light-induced optical waveguide uptapers," Opt. Lett., vol. 18, pp. 1035-1037, July 1993.

[9] S. Shoji and S. Kawata, "Optically-induced growth of fiber patterns into a photopolymerizable resin,” Appl. Phys. Lett., vol. 75, pp. 737-739, Aug. 1999.

[10] _ - "Photofabrication of three-dimensional photonic crystals by multibeam laser interference into a photopolymerizable resin," Appl. Phys. Lett., vol. 76, pp. 2668-2670, May 2000.

[11] D. J. Moss, J. Cannings, M. Faith, S. Madden, P. Kemeny, L. Poladian, F. Ladouceur, J. D. Love, C. V. Poulsen, and O. Leistiko, "Ultra-strong UV written gratings in PECVD grown germanosilicate waveguides," Photosensitive Opt. Mater. Devices, vol. 2998, pp. 142-145, 1997.

[12] M. Fujimaki, S. Shimoto, N. Miyazaki, Y. Ohki, K. S. Seol, and K. Imamura, "Effect of annealing on Ge-doped $\mathrm{SiO}_{2}$ thin films," J. Appl. Phys., vol. 86, pp. 5270-5273, Nov. 1999.

[13] H. Yayama, S. Fujino, K. Morinaga, H. Takebe, D. W. Hewak, and D. N. Payne, "Refractive index dispersion of gallium lanthanum sulfide and oxysulfide glasses," J. Non-Cryst. Solids, vol. 239, pp. 187-191, Oct. 1998.

[14] D. J. Brady, T. Schweizer, J. Wang, and D. W. Hewak, "Minimum loss predictions and measurements in gallium lanthanum sulphide based glasses and fiber," J. Non-Cryst. Solids, vol. 242, pp. 92-98, Dec. 1998.

[15] G. Meltz, W. W. Morey, and W. H. Glenn, "Formation of Bragg gratings in optical fibers by a transverse holographic method," Opt. Lett., vol. 14, pp. 823-825, Aug. 1989.
[16] A. E. Siegman, Lasers. Mill Valley, CA: Univ. Sci. Books, 1986, pp. $627-628$

[17] T. M. Monro, C. M. de Sterke, and L. Poladian, "Analysis of self-written waveguide experiments," J. Opt. Soc. Amer. B, vol. 16, pp. 1680-1685, Oct. 1999.

[18] A. W. Snyder and J. D. Love, Optical Waveguide Theory. London, U.K.: Chapman \& Hall, 1983, p. 7.

[19] K. E. Youden, T. Grevatt, R. W. Eason, H. N. Rutt, R. S. Deol, and G Wylangowski, "Pulsed laser deposition of Ga-La-S chalcogenide glass thin film optical waveguides," Appl. Phys. Lett., vol. 63, pp. 1601-1603, Sept. 1993.

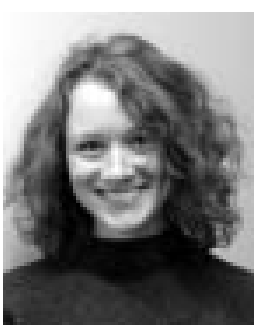

Ami M. Ljungström received the M.S. degree in physics from Göteborg University, Göteborg, Sweden, in 2000. She is currently pursuing the Ph.D. degree at the Optoelectronics Research Centre ORC), University of Southampton, Southampton, U.K. Her topic is experiments and modeling of self-written waveguides in bulk glasses.

Ms. Ljungström is a member of the Optical Society of America (OSA).

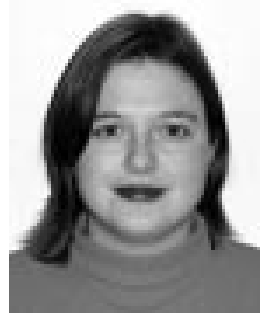

Tanya M. Monro received the Ph.D. degree in selfwritten waveguides from Sydney University, Sydney, Australia.

She currently holds a Royal Society Research Fellowship at the Optoelectronics Research Centre (ORC), University of Southampton, Southampton, U.K. Her research interests include novel waveguides, particularly microstructured or holey fibers and self-written structures.

Dr. Monro is a member of the Optical Society of America (OSA). 